\title{
On a Kinetic Model for Shallow Water Waves
}

\author{
Jens Struckmeier \\ Department of Mathematics \\ University of Kaiserslautern \\ Germany
}

\begin{abstract}
The system of shallow water waves is one of the classical examples for nonlinear, twodimensional conservation laws. The paper investigates a simple kinetic equation depending on a parameter $\varepsilon$ which leads for $\varepsilon \rightarrow 0$ to the system of shallow water waves. The corresponding 'equilibrium' distribution function has a compact support which depends on the eigenvalues of the hyperbolic system. It is shown that this kind of kinetic approach is restricted to a special class of nonlinear conservation laws. The kinetic model is used to develop a simple particle method for the numerical solution of shallow water waves. The particle method can be implemented in a straightforward way and produces in test examples sufficiently accurate results.
\end{abstract}

\section{Introduction}

A well-known example for the relation between a macroscopic and a microscopic description of transport phenomena is given by the Euler equations for equilibrium flows on one side and the Boltzmann equation for rarefied gas flows on the other side.

The Euler equations are defined by a nonlinear system of partial differential equations for the macroscopic quantities density, stream velocity and temperature, whereas the Boltzmann equation is a nonlinear transport equation of the form

$$
\frac{\partial f}{\partial t}+\xi \nabla_{x} f=\frac{1}{\varepsilon} J(f, f)
$$

for the kinetic distribution function $f(t, x, \xi)$.

Using an expansion of the function $f(t, x, \xi)$ with respect to the parameter $\varepsilon$ in the form

$$
f(t, x, \xi)=f_{0}(t, x, \xi)+\varepsilon f_{1}(t, x, \xi)+O\left(\varepsilon^{2}\right)
$$

one can derive the Euler equations using (1) in the singular limit $\varepsilon \rightarrow 0$.

The $0^{\text {th }}$ order of the expansion is given by a Maxwellian $f_{0}$

$$
f_{0}[\rho, u, T]=\frac{\rho}{(2 \pi R T)^{3 / 2}} \exp \left(-\frac{(\xi-u)^{2}}{2 R T}\right)
$$

The function $f_{0}$ is often called equilibrium distribution because the relation

$$
J\left(f_{0}, f_{0}\right)[\rho, u, T]=0
$$

holds for arbitrary $(\rho, u, T)$. 
Several authors have used the relation between the Euler equation and the Boltzmann equation to construct kinetic schemes for Euler equations (see for example references [3],[4] and [7]). For of a general nonlinear scalar conservation law Bäcker and Dressler ([2]) have developed a kinetic distribution function such that the $0^{\text {th }}$ moment of the distribution function gives a solution of the scalar conservation law. A much more elaborated form of this approach can be found in [1].

In the present paper we develop a kinetic model for the nonlinear system of shallow water waves. Furthermore we present a very simple particle method for the numerical solution of shallow water waves. The paper is organized as follows:

In the sequel we give a short description of the system of shallow water waves. A detailed description including many examples of practical relevance can be found in [5]. In section 2 we give the construction of the kinetic model used in the current investigation. Furthermore it is shown in section 3 that this kind of kinetic approach is restricted to a special class of nonlinear twodimensional conservation laws. The particle method for shallow water waves based on the kinetic model is presented in section 4 together with a simple example.

Shallow water waves are described by the system of partial differential equations for the unknown functions $h(t, x)$ (the height of the water) and $u(t, x)$ (the velocity) in the form

$$
\left(\begin{array}{l}
h \\
u
\end{array}\right)_{t}+M(u, h) \cdot\left(\begin{array}{l}
h \\
u
\end{array}\right)_{x}=0
$$

where the matrix $M(U)$ is given by

$$
\left(\begin{array}{ll}
u & h \\
1 & u
\end{array}\right)
$$

The matrix $M(U)$ possesses the two eigenvalues

$$
\lambda^{(1)}(t, x)=u(t, x)-\sqrt{h(t, x)} \quad \lambda^{(2)}(t, x)=u(t, x)+\sqrt{h(t, x)}
$$

If we assume that $h(t, x)>0$ the two eigenvalues are real and distinct and hence the system is strictly hyperbolic.

The characteristic curves are defined by

$$
\dot{x}^{(1)}=\lambda^{(1)} \quad \dot{x}^{(2)}=\lambda^{(2)}
$$

Along the characteristic curves the Riemann invariants

$$
r^{(1)}=u-2 \sqrt{h} \quad r^{(2)}=u+2 \sqrt{h}
$$

remain constant.

By introducing the conservation variables $(v, w)$ we can write the system in the form

$$
\left(\begin{array}{c}
v \\
w
\end{array}\right)_{t}+\left(\begin{array}{c}
w \\
\frac{1}{2} v^{2}+\frac{w^{2}}{v}
\end{array}\right)_{x}=0
$$

where

$$
v(t, x)=h(t, x) \quad w(t, x)=(h \cdot u)(t, x)
$$

For simplicity we denote the flux of $w$ by $f[v, w]$.

$$
f[v, w](t, x)=\left(\frac{1}{2} v^{2}+\frac{w^{2}}{v}\right)(t, x)
$$

Concerning the theory of hyperbolic systems we refer to reference [5]. 


\section{A Kinetic Equation for Shallow Water Waves}

In this section we construct a scalar kinetic equation in the form

$$
G_{t}+\xi \cdot G_{x}=\mathcal{P}_{\varepsilon}[G] \quad \varepsilon>0
$$

which should lead formally to the system of shallow water waves in the singular limit $\varepsilon \rightarrow 0$. The relation between the conservation variables of (2) and the kinetic distribution function $G(t, x, v)$ should be given by

$$
\begin{aligned}
v(t, x) & =\int_{\boldsymbol{R}} G(t, x, \xi) \mathrm{d} \xi \\
w(t, x) & =\int_{\boldsymbol{R}} \xi \cdot G(t, x, \xi) \mathrm{d} \xi
\end{aligned}
$$

$v$ and $w$ are the $0^{\text {th }}$ and $1^{\text {st }}$ moment of the $G$.

Furthermore - to get the correct singular limit - we force the operator $\mathcal{P}_{\varepsilon}$ to have the properties

$$
\begin{array}{rlrl}
\varepsilon \mathcal{P}_{\varepsilon}[G] & =O(1) \quad \text { for } \quad \varepsilon \rightarrow 0 \\
\int_{\mathbb{R}} \mathcal{P}_{\varepsilon}[G] \mathrm{d} \xi & =0 & \\
\int_{\boldsymbol{R}} \xi \mathcal{P}_{\varepsilon}[G] \mathrm{d} \xi & =0 \quad \forall \varepsilon>0
\end{array}
$$

To ensure a consistency between the descriptions (2) and (3) the flux function $f[v, w]$ must be given by the second moment of $G$

$$
f[v, w](t, x)=\int_{\boldsymbol{R}} \xi^{2} \cdot G(t, x, \xi) \mathrm{d} \xi
$$

It follows from a straighforward calculus that in the singular limit the first and second moment behave according to the system of shallow water waves.

Besides this formal description one has to check if it is possible to define a kinetic function $G$ such that the relations (4), (5) and (6) hold. In analogy to the Euler equations (see reference [4]) we assume that $G$ depends only implicitly on $(t, x)$ and take an ansatz in the form

$$
G(t, x, \xi)=\left\{\begin{array}{cc}
g(\gamma-\xi) & \alpha \leq \xi \leq \gamma \\
g(\xi-\gamma) & \gamma \leq \xi \leq \beta \\
0 & \text { elsewhere }
\end{array}\right.
$$

where $\gamma=(\alpha+\beta) / 2$ and $(\alpha, \beta)=(\alpha, \beta)(t, x)$.

Taking this ansatz the operator $\mathcal{P}_{\varepsilon}$ may be defined by

$$
\mathcal{P}_{\varepsilon}[G]=\frac{1}{\varepsilon}(G-\bar{G})
$$

where $\bar{G}$ denotes the projection of a arbitrary function $G$ to the ansatz (7). The operator $\mathcal{P}_{\varepsilon}$ plays the role of a relaxation to the 'equilibrium' distribution given by (7) with relaxation 
parameter $\varepsilon$.

Assuming the ansatz (7) one has to define the free parameters $\alpha, \beta$ and $g$ in order to get a consistency between the two approaches.

By differentiating (4),(5) and (6) with respect to $\alpha$ and $\beta$ one gets

$$
\begin{aligned}
& v_{\alpha}=-g(\gamma-\alpha) \\
& w_{\alpha}=-\alpha \cdot g(\gamma-\alpha)-\int_{\gamma}^{\beta}(\xi-\gamma) g^{\prime}(\xi-\gamma) \mathrm{d} \xi \\
& f_{\alpha}=-\alpha^{2} \cdot g(\gamma-\alpha)-2 \cdot \gamma \cdot \int_{\gamma}^{\beta}(\xi-\gamma) g^{\prime}(\xi-\gamma) \mathrm{d} \xi \\
& v_{\beta}=g(\beta-\gamma) \\
& w_{\beta}=\beta \cdot g(\beta-\gamma)-\int_{\gamma}^{\beta}(\xi-\gamma) g^{\prime}(\xi-\gamma) \mathrm{d} \xi \\
& f_{\beta}=\beta^{2} \cdot g(\beta-\gamma)-2 \cdot \gamma \cdot \int_{\gamma}^{\beta}(\xi-\gamma) g^{\prime}(\xi-\gamma) \mathrm{d} \xi
\end{aligned}
$$

Because $f$ is a function of $v$ and $w$ we have

$$
f_{\alpha}=f_{v} \cdot v_{\alpha}+f_{w} \cdot w_{\alpha} \quad f_{\beta}=f_{v} \cdot v_{\beta}+f_{w} \cdot w_{\beta}
$$

and using (11) and (14) yields

$$
\begin{aligned}
& \left(\alpha^{2}-f_{w} \cdot \alpha-f_{v}\right) \cdot g\left(\frac{\beta-\alpha}{2}\right)=\left(f_{w}-2 \cdot \gamma\right) \cdot \int_{\gamma}^{\beta}(\xi-\gamma) g^{\prime}(\xi-\gamma) \mathrm{d} \xi \\
& \left(\beta^{2}-f_{w} \cdot \beta-f_{v}\right) \cdot g\left(\frac{\beta-\alpha}{2}\right)=-\left(f_{w}-2 \cdot \gamma\right) \cdot \int_{\gamma}^{\beta}(\xi-\gamma) g^{\prime}(\xi-\gamma) \mathrm{d} \xi
\end{aligned}
$$

The quadratic equation on the left hand side of (16) and (17) is exactly the characteristic equation for the eigenvalues of the hyperbolic system (2) and by defining $\alpha$ and $\beta$ as the two eigenvalues it follows directly

$$
f_{w}-2 \cdot \gamma=0
$$

Lemma 1 If $\alpha$ and $\beta$ are the eigenvalues of the system (2) then equations (16) and (17) hold.

Furthermore, to get a consistency between (2) and (3) the compatibility condition between $v_{\alpha}$ and $w_{\alpha}$, respectively $v_{\beta}$ and $w_{\beta}$ must be satisfied. The condition are given by substituting (9) in (10), respectively (12) in (13):

$$
w_{\lambda}=\lambda \cdot v_{\lambda}-\int_{\gamma}^{\beta}(\xi-\gamma) g^{\prime}(\xi-\gamma) \mathrm{d} \xi
$$


for $\lambda \in\{\alpha, \beta\}$.

The compatibility conditions may be fulfilled by playing with the shape of the function $g$ : Introducing the transformation

$$
\gamma=\frac{\alpha+\beta}{2} \quad \eta=\frac{\beta-\alpha}{2}
$$

and using partial integration in (18) we get the following two equations:

$$
\begin{aligned}
& \int_{0}^{\eta} g(x) \mathrm{d} x=\frac{1}{2}\left(w_{\gamma}-\gamma \cdot v_{\gamma}\right)-\frac{1}{2}\left(w_{\eta}-\gamma \cdot v_{\eta}\right) \\
& \int_{0}^{\eta} g(x) \mathrm{d} x=\frac{1}{2}\left(w_{\gamma}-\gamma \cdot v_{\gamma}\right)+\frac{1}{2}\left(w_{\eta}-\gamma \cdot v_{\eta}\right)
\end{aligned}
$$

It is obvious that this system can only have a solution if

$$
w_{\eta}=\gamma \cdot v_{\eta}
$$

If we assume that (19) holds we get

$$
g(\eta)=\frac{1}{2}\left(w_{\gamma \eta}-\gamma \cdot v_{\gamma \eta}\right)
$$

which defines the shape of $g$.

\section{Remark 1}

One has to be careful: Equation (20) is valid only if $w_{\gamma}-\gamma \cdot v_{\gamma}$ does not depend on $\gamma$, otherwise the system given above has no solution.

For the system of shallow water waves it holds that

$$
v=\eta^{2} \quad w=\gamma \cdot \eta^{2}
$$

and equation (20) becomes

$$
g(\eta)=\eta
$$

The result can be summarized in the following lemma.

Lemma 2 Given the system of shallow water waves in the form (2). Then it is possible to construct a kinetic function which solves the equation

$$
G_{t}+\xi \cdot G_{x}=\mathcal{P}_{\varepsilon}[G]
$$

where the quantities $v, w$ and $f[v, w]$ are given by the moments of the function $G(t, x, \xi)$ in the form (4),(5) and (6).

The function $G(t, x, \xi)$ depends only implicitly on $t$ and $x$ and is given by

$$
G(t, x, \xi)=\left\{\begin{array}{cc}
\gamma-\xi & \alpha \leq \xi \leq \gamma \\
\xi-\gamma & \gamma \leq \xi \leq \beta \\
0 & \text { elsewhere }
\end{array}\right.
$$

where $\alpha$ and $\beta$ are the two eigenvalues of (2) and $\gamma=(\alpha+\beta) / 2$. For the singular limit $\varepsilon \rightarrow 0$ the system of shallow water waves can be formally derived from the scalar kinetic equation. 


\section{Remark 2}

The relation between the two descriptions (2) and (3) can also be explained by defining a set $M_{e q}$ of distribution functions with

$$
M_{e q}=\left\{G(\xi) \in L^{1}(I R): \exists v, w|\bar{G}[v, w]-G|_{\infty}=0\right\}
$$

where $\bar{G}$ denotes a function in the form given by (21).

Starting from the scalar kinetic equation

$$
G_{t}+\xi G_{x}=\mathcal{P}_{\varepsilon}[G] \quad \mathcal{P}_{\varepsilon}[G]=\frac{1}{\varepsilon}(\bar{G}-G)
$$

with initial condition $\stackrel{\circ}{G}(x, \xi) \in M_{e q}$ for all $x \in I R$ we consider the time evolution of the distribution function $G(t, x, v)$ under the constraint

$$
G(t, x, \xi) \in M_{e q} \quad \forall(t, x) \in I R_{+} \times I R
$$

The parameters $v(t, x)$ and $w(t, x)$ which describe the function $G(t, x, \xi) \in M_{e q}$ are a solution of the macroscopic system (2).

\section{The General Case}

We now investigate a general system of the form

$$
\left(\begin{array}{c}
v \\
w
\end{array}\right)_{t}+\left(\begin{array}{c}
w \\
f[v, w]
\end{array}\right)_{x}=0
$$

where $f[v, w]$ is an arbitrary function of $(v, w)$ and we try to construct - as in the case of shallow water waves - a scalar kinetic equation in the form

$$
G_{t}+\xi \cdot G_{x}=\mathcal{P}_{\varepsilon}[G]
$$

where

$$
G(t, x, \xi)=\left\{\begin{array}{cc}
g(\gamma-\xi) & \alpha \leq \xi \leq \gamma \\
g(\xi-\gamma) & \gamma \leq \xi \leq \beta \\
0 & \text { elsewhere }
\end{array}\right.
$$

Again $v(t, x)$ and $w(t, x)$ should be the $0^{t h}$ and $1^{s t}$ moments of $G$ and $\mathcal{P}_{\varepsilon}$ should be the relaxation term of an arbitrary function $G$ to the form (24).

Using the same techniques as in the section above we choose the bounds $\alpha(t, x)$ and $\beta(t, x)$ as the eigenvalues of the general system (22), i.e.

$$
\begin{aligned}
& \alpha(t, x)=\frac{f_{w}}{2}-\frac{1}{2} \cdot\left(f_{w}^{2}+4 \cdot f_{v}\right)^{1 / 2} \\
& \beta(t, x)=\frac{f_{w}}{2}+\frac{1}{2} \cdot\left(f_{w}^{2}+4 \cdot f_{v}\right)^{1 / 2}
\end{aligned}
$$

It is obvious that the transformation from $(v, w) \rightarrow(\alpha, \beta)$ must be local invertible which can only hold if $f[v, w]$ is a strictly nonlinear function of both $v$ and $w$. 
We assume that $(v, w) \rightarrow(\alpha, \beta)$ is everywhere local invertible.

The first condition to find consistency using the ansatz (24) is

$$
w_{\eta}=\gamma \cdot v_{\eta}
$$

This requirement can be transformed into a condition on $f(v, w)-f_{w}$ must be a solution of a Burger's like equation.

Lemma 3 A necessary condition to get a consistency between the system (22) and the scalar kinetic equation (23) with the ansatz (24) is that $f_{w}$ is a solution of the scalar conservation law

$$
h_{v}+\left(\frac{h^{2}}{4}\right)_{w}=0
$$

with an arbitrary initial condition $h(\stackrel{0}{v}, w)=\stackrel{\circ}{h}(w)$.

Proof We define $\stackrel{o}{v}$ such that $f_{w}(\stackrel{o}{v}, w)<\infty$ and $\stackrel{o}{h}=f(\stackrel{o}{v}, w)$.

Furthermore we assume that $f_{w}$ is a solution of

$$
h_{v}+\left(\frac{h^{2}}{4}\right)_{w}=0
$$

with initial condition $f_{w}(\stackrel{o}{v}, w)=\stackrel{o}{h}(w)$.

Consider the transformation of variables $T(\gamma, \eta)=(v(\gamma, \eta), w(\gamma, \eta))$, then

$$
D T=\left(\begin{array}{ll}
v_{\gamma} & w_{\gamma} \\
v_{\eta} & w_{\eta}
\end{array}\right)
$$

By the inverse function theorem it follows that $D T \cdot D T^{-1}=i d_{\mathbb{R}^{2}}$ and

$$
D T^{-1}=\left(\begin{array}{cc}
\gamma_{v} & \eta_{v} \\
\gamma_{w} & \eta_{w}
\end{array}\right)=(D T)^{-1}=\frac{1}{c}\left(\begin{array}{cc}
w_{\eta} & -w_{\gamma} \\
-v_{\eta} & v_{\gamma}
\end{array}\right)
$$

where $c=\operatorname{det}(D T)$.

Therefore equation (26) can be written as

$$
\gamma_{v}+\gamma \cdot \gamma_{w}=0
$$

and relation (28) yields

$$
w_{\eta}-\gamma \cdot w_{\eta}=0
$$

Corollary $1 \quad f_{w}$ is a solution of equation (25) if and only if $\eta_{w}=0$

Proof We have

$$
\eta=\frac{1}{2} \cdot\left(f_{w}^{2}+4 f_{v}\right)^{1 / 2}
$$

Differentiating with respect to $w$ yields

$$
\eta_{w}=\frac{1}{2} \cdot\left(f_{w}^{2}+4 f_{v}\right)^{-1 / 2} \cdot\left(\frac{f_{w}}{2} f_{w w}+f_{w v}\right)
$$


By substituting $h=f_{w}$ equation (32) gets

$$
\left.\eta_{w}=\frac{1}{2} \cdot\left(f_{w}^{2}+4 f_{v}\right)^{-1 / 2} \cdot\left(h_{v}+\left(\frac{h^{2}}{4}\right) w\right)\right)
$$

The second consistency condition for a general system is given by

$$
w_{\gamma}-\gamma \cdot v_{\eta}=p(\eta)
$$

where $p$ is an arbitrary function of $\eta$.

Lemma 4 Suppose that $\eta_{w}=0$, then $w_{\gamma}-\gamma \cdot v_{\eta}$ is independent of $\gamma$ if there exists a function $p$ such that

$$
\frac{1}{f_{w w}} \cdot p\left(\frac{1}{2}\left(f_{w}^{2}+4 \cdot f_{v}\right)^{1 / 2}\right)=c
$$

where $c$ is an arbitrary constant.

Proof By substituting the variables $(v, w) \rightarrow(\gamma, \eta)$ and using $D T \cdot D\left(T^{-1}\right)=\mathrm{id}_{\boldsymbol{R}^{2}}$ equation (34) becomes

$$
-\frac{1}{c} \cdot\left(\eta_{v}+\gamma \cdot \eta_{w}\right)=p(\eta)
$$

where $c=\gamma_{v} \cdot \eta_{w}-\eta_{v} \cdot \gamma_{w}$.

By the assumption $\eta_{w}=0$ one gets

$$
\frac{1}{\gamma_{w}}=p(\eta)
$$

Using $\gamma=\frac{f_{w}}{2}$ yields

$$
\frac{1}{f_{w w}}=p\left(\frac{1}{2}\left(f_{w}^{2}+4 \cdot f_{v}\right)^{1 / 2}\right)
$$

Now we are able to formulate a consistency condition between the system (22) and the scalar kinetic equation (23) with the ansatz (24).

Theorem 1 The scalar kinetic equation

$$
G_{t}+\xi \cdot G_{x}=\mathcal{P}_{\varepsilon}[G]
$$

with the ansatz

$$
G(t, x, \xi)=\left\{\begin{array}{cc}
g(\gamma-\xi) & \alpha \leq \xi \leq \gamma \\
g(\xi-\gamma) & \gamma \leq \xi \leq \beta \\
0 & \text { elsewhere }
\end{array}\right.
$$

leads to a consistent description for a general system of nonlinear conservation laws

$$
\left(\begin{array}{c}
v \\
w
\end{array}\right)_{t}+\left(\begin{array}{c}
w \\
f(v, w)
\end{array}\right)_{x}=0
$$

if and only if the function $f[v, w]$ is given by

$$
f[v, w]=\frac{w^{2}}{c_{1}+v}+c_{2} \cdot \frac{w}{c_{1}+v}+c_{3}(v)
$$

where $c_{1}, c_{2}$ are arbitrary constants and $c_{3}(v)$ is an arbitrary function of $v$. 
Proof Using Corollary 1 we have $\eta_{w}=0$, which means that $\eta$ depends only on $v$. Therefore by Lemma 4 we have the relation

$$
f_{w w}=p(v)
$$

where $p(v)$ is an arbitrary function of $v$.

Then it follows directly that

$$
f(v, w)=a(v) \cdot w^{2}+b(v) \cdot w+c(v)
$$

Now

$$
\eta=\frac{1}{2} \cdot\left(f_{w}^{2}+4 \cdot f_{v}\right)^{1 / 2}
$$

must be a function of $v$ because $\eta_{w}=0$.

Substituting (35) into (36) yields

$$
\eta=\left[\left(a^{\prime}(v)+a^{2}(v)\right) \cdot w^{2}+\left(b^{\prime}(v)+a(v) b(v)\right) \cdot w+\frac{b^{2}(v)}{4}+c^{\prime}(v)\right]^{1 / 2}
$$

Because the right hand side must be independent of $w$ the differential equations

$$
a^{\prime}(v)=a^{2}(v) \quad b^{\prime}(v)=-a(v) b(v)
$$

must hold.

By integration we get

$$
a(v)=\frac{1}{c_{1}+v} \quad b(v)=\frac{c_{2}}{c_{1}+v}
$$

where $c_{1}, c_{2}$ are arbitrary constants.

\section{Remark 3}

The shape of the 'equilibrium' distribution function is defined by the equation (20).

\section{A Particle Method for Shallow Water Waves}

The scalar kinetic equation developed in section 2 can be used to construct a simple particle method for the numerical solution of shallow water waves.

The technique is very similar to particle methods for Euler equations which can be found for example in the references [3] and [4]. Concerning the general concept of particle methods we refer to [6] where the reader may find a much more elaborated particle method for the full Boltzmann equation.

The general idea of a particle method for a nonlinear transport equation is the following: The kinetic distribution function $G(t, x, \xi)$ is considered as the density of a continuous measure $\mu_{t}$ on the phase space $\Omega \times I R$ which varies with time $t$ according to the given transport equation. Using the concept of weak convergence of measures one can approximate the given continuous measure $\mu_{t}$ by a sum of discrete measures in the form

$$
\mu_{N}(t)=\sum_{i=1}^{N} \delta_{x_{i}} \times \delta_{v_{i}}
$$


such that the finite pointset $\mu_{N}(t)$ converges for $N \rightarrow \infty$ weakly to $\mu_{t}$ - which means that

$$
\left\langle\phi, \mu_{N}(t)\right\rangle \stackrel{w}{\longrightarrow}\left\langle\Phi, \mu_{t}\right\rangle \quad \forall \Phi \in \mathcal{C}_{0}^{b}(I R) t \geq 0
$$

The main task is to construct a time evolution of the finite pointset such that the above relation holds for all $t>0$.

In the case of the scalar kinetic equation given by (3) the construction of a time evolution is very simple:

We consider first a time discretized form of the equation (3)

$$
G(\Delta t, x, \xi)=G(0, x, \xi)+\Delta t \mathcal{P}_{\varepsilon}[G(0, x, \xi)]
$$

The density function $G$ is approximated by a sum of step functions based on a gridstructure of the spatial domain $\Omega$

$$
G(n \Delta t)=\sum_{i=1}^{m} G_{m}(n \Delta t, \xi)
$$

where

$$
\Omega=\bigcup_{i=1, \ldots, m}^{\bullet} \mathcal{Z}_{i} \quad \mathcal{Z}_{i} \in \Omega \quad \mathcal{Z}_{i} \cap \mathcal{Z} j=\emptyset \quad i \neq j
$$

The operator $\mathcal{P}_{\varepsilon}$ of the kinetic equation operates as a relaxation term to the 'equilibrium' distribution given by (7). Using the discretized equation and a splitting method for the time evolution the particle method - in the limit $\varepsilon \rightarrow 0$ - will consists of the following steps:

1) Given the initial condition $G(0, x, \xi)$ of equation (3).

Approximate the function $G(0, x, \xi)$ by a discrete set of particles $\mu_{N}=\left(x_{i}, v_{i}\right)_{i=1, \ldots, N}$ such that

$$
\mu_{N} \stackrel{w}{\longrightarrow} \sum_{i=1}^{m} \stackrel{o}{\mu}_{i}
$$

where $\stackrel{\circ}{\mu}$ is given by

$$
\stackrel{\circ}{\mu_{i}}=\int_{\mathcal{Z}_{i}} G(0, x, \xi) \mathrm{d} x \quad \forall i=1, \ldots, m
$$

2) Perform the translation of the points $\left(x_{i}\right)_{i=1, \ldots, N}$

$$
x_{i}^{\prime}=x_{i}+\Delta t v_{i} \quad \forall i=1, \ldots, N
$$

3) Sample the finite pointset $\left(x_{i}^{\prime}, v_{i}\right)_{i=1, \ldots, N}$ according to the given gridstructure and modify - in every cell - the given point set with respect to the ansatz.

\section{Remark 4}

1) By the use of a splitting method the time evolution is divided in the steps 2) and 3).

2) In the limit $\varepsilon \rightarrow 0$ the operator $\mathcal{P}_{\varepsilon}$ acts as a projection of the given particle distribution to the 'equilibrium' state given by (7). 
3) The generation of a point approximation of the initial condition and the projection with respect to (21) is almost trivial because the shape of the function $G$ is very simple.

\section{Example 1}

We consider the system

$$
\left(\begin{array}{l}
h \\
u
\end{array}\right)_{t}+\left(\begin{array}{ll}
u & h \\
1 & u
\end{array}\right) \cdot\left(\begin{array}{l}
h \\
u
\end{array}\right)_{x}=0
$$

together with the initial conditions

$$
\stackrel{\circ}{h}(x)=1 \quad \stackrel{\circ}{u}(x)=x
$$

The problem has a classical solution for all $t>0$ given by

$$
h(t, x)=\frac{1}{1+t} \quad u(t, x)=\frac{x}{1+t}
$$

The characteristic curves $x^{(1)}(t)$ and $x^{(2)}(t)$ - illustrated in figure 1 - are defined by the equations

$$
\begin{aligned}
& \dot{x}^{(1)}=\frac{x^{(1)}}{1+t}-\sqrt{\frac{1}{1+t}} \quad \dot{x}^{(2)}=\frac{x^{(2)}}{1+t}+\sqrt{\frac{1}{1+t}} \\
& \text { t-axis }
\end{aligned}
$$

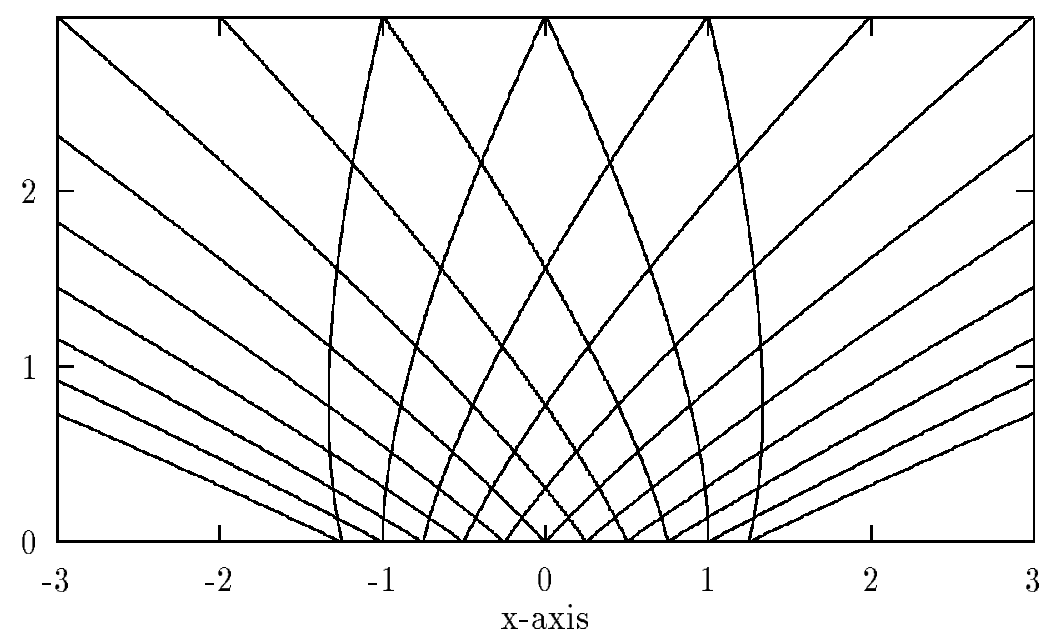

Fig. 1: Characteristic Curves $x^{(1)}(t)$ and $x^{(2)}(t)$

For the implementation of the particle method we consider the spatial domain $[-1,1]$ over the time interval $[0,3]$.

Because it is necessary to prescribe artificial boundary conditions at the points $x_{1}=-1$ and $x_{2}=1$ the ingoing fluxes are modelized using the exact solution. Particles which will cross this points in the direction of the outer normal are deleted.

For the particle method the bounded spatial domain is divided into a regular gridstructure with parameter $\Delta x=0.1$. The time discretization parameter $\Delta t$ is given by 0.05 . At 
time $t=0$ the kinetic distribution function which is homogenized over a single cell is approximated using 500 discrete points. In order to reduce the fluctuations in the numerical results 50 independent samplings are used. The results are obtained on a HP 9000/710 workstation with a CPU time of about 160 seconds.

The numerical results given in figure 2 and 3 show a comparison between the numerical solution - plotted with points - and the exact solution - plotted with lines - at various discrete times $t_{n}=n \Delta t$.

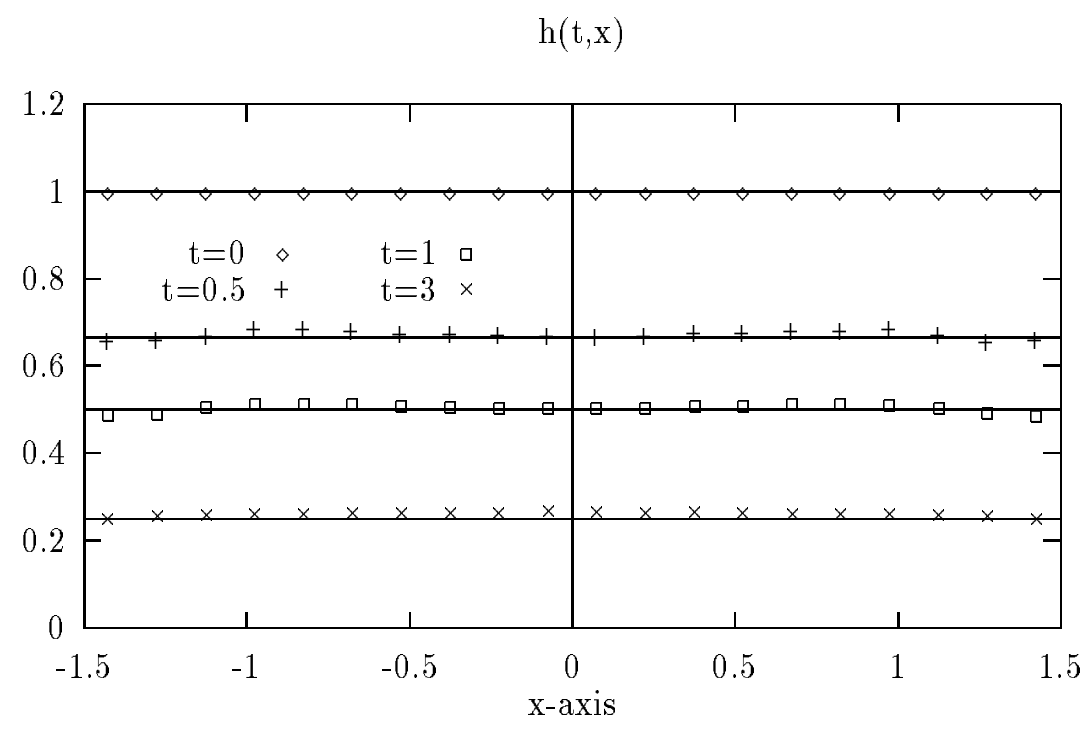

Fig. 2: Function $h(t, x)$ at different times $t_{n}$.

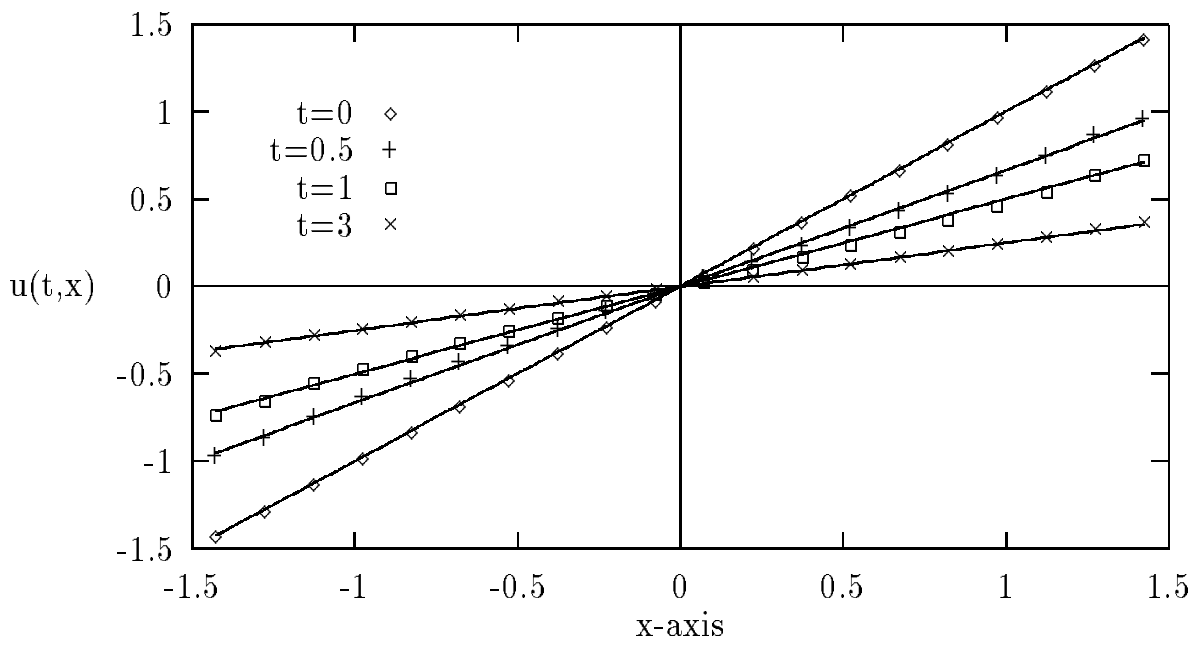

Fig. 3: Function $u(t, x)$ at different times $t_{n}$.

\section{Example 2}

The following example is the so-called dam-breaking problem. We consider this problem 
in terms of the conservation variables $v(t, x)$ and $w(t, x)$.

The initial conditions are given by

$$
\stackrel{o}{v}(x)=\left\{\begin{array}{ll}
1 & x<0 \\
0 & x \geq 0
\end{array} \quad \stackrel{o}{w}(x)=0\right.
$$

The problem has a weak solution in the form

$$
v(x, t)=\left\{\begin{array}{cl}
1 & x<-t \\
\frac{1}{9}\left(2-\frac{x}{t}\right)^{2} & -t \leq x \geq 2 t \\
0 & 2 t<x
\end{array}\right.
$$

and

$$
w(t, x)=\left\{\begin{array}{cl}
0 & x<-t \\
\frac{2}{27}\left(\frac{x}{t}+1\right)\left(2-\frac{x}{t}\right)^{2} & -t \leq x \geq 2 t \\
0 & 2 t<x
\end{array}\right.
$$

The numerical solution is calculated in the spatial domain $[-1.2,2.4]$ where again the fluxes at the artificial boundaries are given by the exact solution.

The cell size is given by $\Delta x=0.05$ and we use a time discretization $\Delta t=0.005$. The initial distribution is approximated by 800 points per cell and 20 independent runs are used to calculate the solution.

The computational costs for this example are 580 seconds.

The agreement between the numerical and exact solution is quite good in the positive $x$-region. In the negative $x$-region the sharp edge at the point $x=-t$ is smeared out.

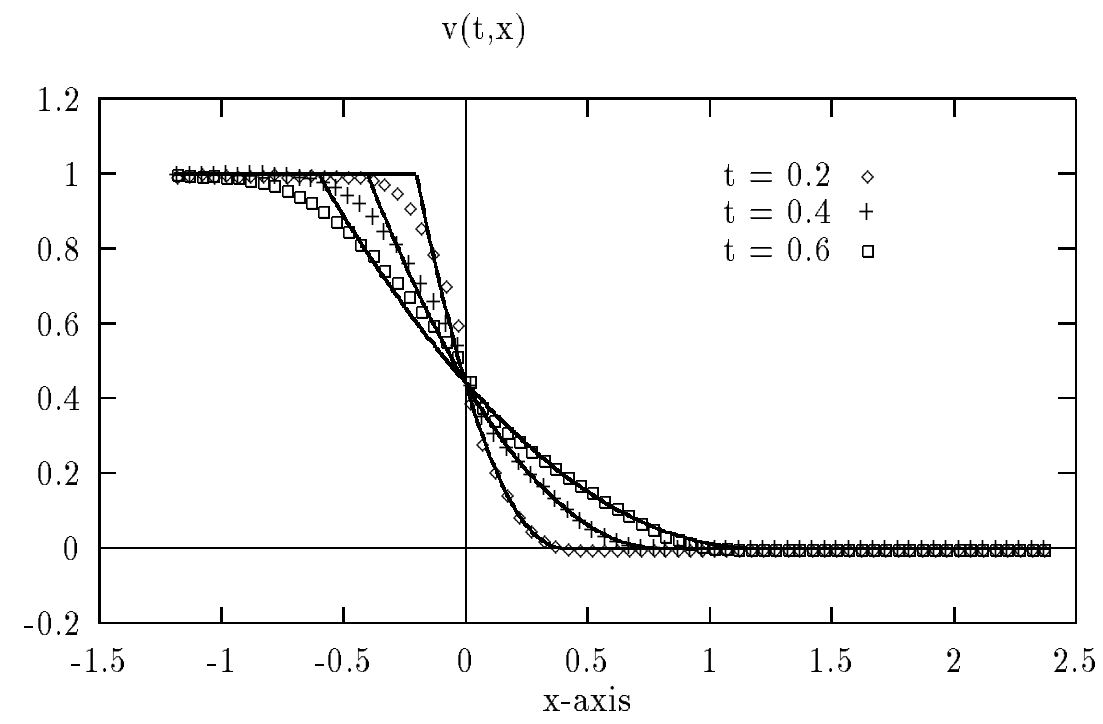

Fig. 4: Function $v(t, x)$ at different times $t_{n}$. 


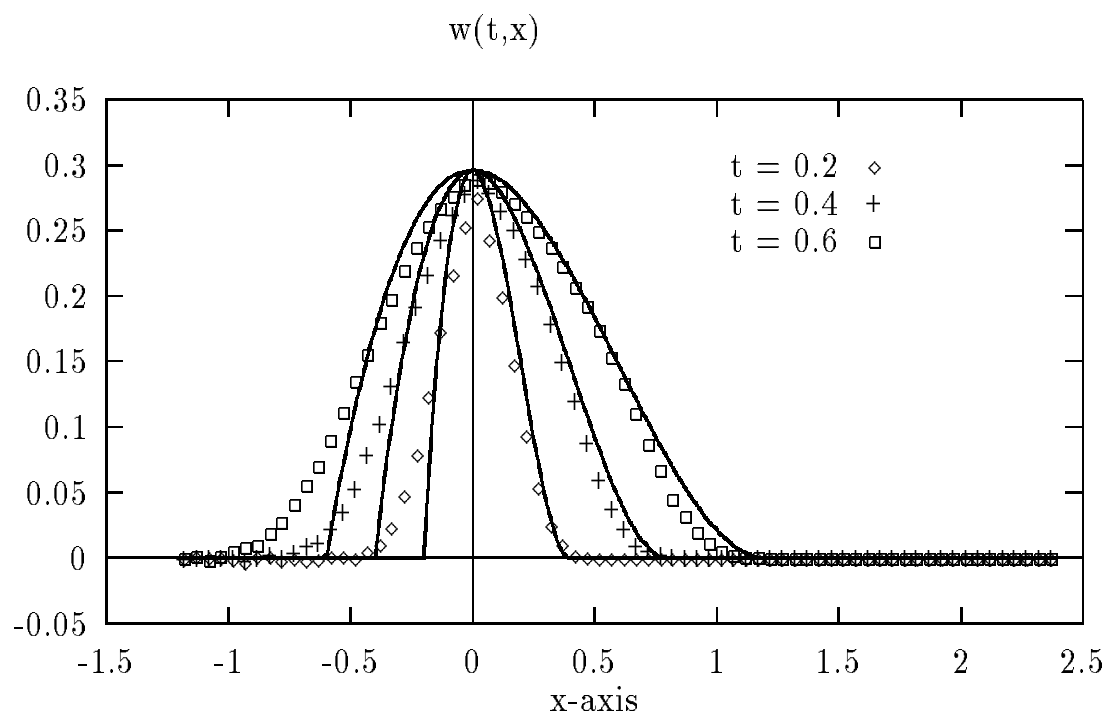

Fig. 5: Function $w(t, x)$ at different times $t_{n}$.

\section{References}

[1] Bäcker, M.:

Kinetische Verfahren für nichtlineare skalare Erhaltungsgleichungen, $\mathrm{PhD}$ Thesis, University Kaiserslautern (1993).

[2] Bäcker, M. and Dressler, K.:

A Kinetic Method for Strictly Nonlinear Conservation Laws, Journal of Appl. Math. \& Phys. (ZAMP), 48, 243-256 (1991).

[3] Deshpande, S.M.:

A Second-Order Accurate Kinetic-Theory Method for Inviscid Compressible Flows, NASA Technical Paper 2613 (1986).

[4] Kaniel, S.:

A Kinetic Model for the Compressible Flow Equations, Indiana University Math. Journal, 37, $537-563$ (1988).

[5] Kervokian, J.:

Partial Differential Equations - Analytical Solution Techniques, Wadsworth (1990).

[6] Neunzert, H. and Struckmeier, J.:

The Finite Pointset Method for hypersonic flows in the rarefied regime, in: Bertin, J.J; Glowinski, R.; Periaux, J. (Eds.), Hypersonic Flows, Volume III, Birkhäuser (1992).

[7] Pullin, D.I.:

Direct Simulation Methods for Compressible Inviscid Ideal-Gas Flow, J. Comp. Physics 34, 231-244 (1980). 